Pathologe $2020 \cdot 41: 207-208$

https://doi.org/10.1007/s00292-020-00788-7

(c) Springer Medizin Verlag GmbH, ein Teil von Springer Nature 2020

Check for
updates

G. Staab' $\cdot$ K. W. Schmid

'Redaktion „Der Pathologe“, Fachzeitschriften Medizin, Springer Medizin Verlag GmbH, Heidelberg, Deutschland

${ }^{2}$ Institut für Pathologie, Universitätsklinikum Essen, Universität Duisburg-Essen, Essen, Deutschland

\title{
Open Access publizieren dank DEAL-Vereinbarung
}

\section{Geben Sie Ihren wissenschaftlichen Beiträgen die größtmögliche Verbreitung}

\section{Was bedeutet das für Sie?}

ab Anfang diesen Jahres lohnt es sich noch mehr Ihre Originalarbeiten, Übersichten aber auch Kasuistiken in „Der Pathologe“ zu publizieren. Neben der großen Reichweite im Abonnentenkreis der Zeitschrift, die einen Großteil der Pathologen/innen in Deutschland erreicht, haben Sie seit Jahresanfang auch die Möglichkeit, Ihre frei eingereichten Arbeiten über das Projekt DEAL Open-Access zu publizieren. Im Folgenden möchten wir Sie über dessen Rahmenbedingungen informieren.

Was ist das Projekt DEAL?

Das Projekt DEAL ([1]; - Abb. 1) ist eine Vereinbarung der Deutschen Wissenschaftsorganisationen und bisher zwei Verlagen mit den Zielen, den $\mathrm{Zu}$ griff auf Verlagsinhalte $\mathrm{zu}$ erleichtern sowie Open-Access Publikationen zu fördern. Am 08.01.2020 wurde in diesem Zusammenhang eine Vereinbarung mit SpringerNature getroffen, welche fast alle Zeitschriften des Verlags umfasst und rückwirkend zum 01.01.2020 für drei Jahre greift.

In dieser Vereinbarung ist auch unsere Zeitschrift „Der Pathologe“ involviert, worüber wir uns sehr freuen.

Dies ist ein verlagsseitiges Editorial, welches in ähnlicher Form in mehreren Zeitschriften des SpringerMedizinVerlags erscheint.
$\mathrm{Ab}$ sofort haben korrespondierende $\mathrm{Au}$ toren der teilnehmenden Einrichtungen ([2]; - Abb. 2) die Möglichkeit ohne die Problematik der Finanzierung ausgewählte wissenschaftliche Beiträge in "Der Pathologe“ Open-Access zu publizieren und so mehr Leser als jemals zuvor zu erreichen - für „Der Pathologe“ eine einmalige Option. Da OpenAccess Beiträge durchschnittlich 2,5mal häufiger gelesen und rund $30 \%$ häufiger zitiert werden, sichert diese Option eine größere Sichtbarkeit Ihres Artikels, aber auch der Zeitschrift.

Beiträge von korrespondierenden Autoren, welche keiner teilnehmenden Einrichtung angehören, können selbstverständlich weiterhin auf dem üblichen Weg (Open Choice) Open-Access publiziert werden.

\section{Welche Beiträge werden von DEAL abgedeckt?}

Mit der Vereinbarung DEAL möchten die Unterzeichner die Transformation in

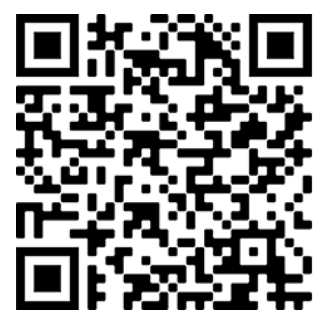

Abb. $1 \Delta$ QR-Code zu ProjektDEAL.Informieren Sie sich hier der Wissenschaft unterstützen und wissenschaftliche Arbeiten aus den Universitäten und Forschungseinrichtungen frei zugänglich machen. Gemäß des Vertrags, den Sie unter [3] vollständig einsehen können,, werden in „Der Pathologe" die Artikel abgedeckt, die frei eingereicht werden, also Originalarbeiten und Kasuistiken oder frei eingereichte (Kurz-) Übersichten.

Die Zeitschrift „Der Pathologe“ verfolgt - unter anderem mit dem regelmäBigen Leitthema, den CME-Beiträgen und den diversen Rubriken - hauptsächlich einen edukativen Ansatz. Diese Beiträge fallen definitionsgemäß nicht unter die DEAL-Vereinbarung,können aber natürlich weiterhin auf dem üblichen Weg (Open Choice) Open-Access publiziert werden.

Wir glauben, dass DEAL ein sehr guter Grund ist in „Der Pathologe“ deutschsprachig $\mathrm{zu}$ publizieren und laden Sie daher heute mit besonderem Nachdruck ein, Ihre entsprechenden freien Beiträge einzureichen. Bitte nutzen Sie dafür den Editorial Manager (www. editorialmanager.com/nore). Sie finden

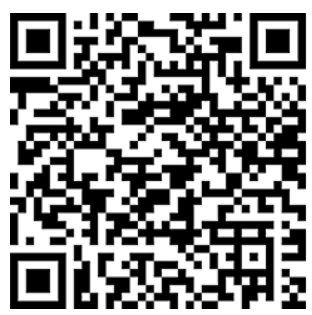

Abb. $2 \triangle$ Alle teilnehmenden Einrichtungen finden Sie hinter diesem QR-Code 
alle Informationen zu den Artikeltypen und den formalen Rahmenbedingungen unter "Submissionguidelines“ auf der Zeitschriftenhomepage [4]. Nutzen Sie DEAL, um die die Sichtbarkeit Ihrer Arbeit zu erhöhen.

\section{Korrespondenzadresse}

\section{G. Staab}

Redaktion „Der Pathologe“, Fachzeitschriften

Medizin, Springer Medizin Verlag GmbH

Tiergartenstr. 17, 69121 Heidelberg,

Deutschland

gabriele.staab@springer.com

\section{Prof. Dr. med. K. W. Schmid}

Institut für Pathologie, Universitätsklinikum

Essen, Universität Duisburg-Essen

Hufelandstr. 55, 45147 Essen, Deutschland

kw.schmid@uk-essen.de

Interessenkonflikt. G. Staab und K.W. Schmid geben an, dass kein Interessenkonflikt besteht.

\section{Literatur}

1. Projekt DEAL (2020) Webpräsenz. https:// www.projekt-deal.de/aktuelles/. Zugegriffen: 16.04.2020

2. Projekt DEAL (2020) FAQ für teilnehmende Einrichtungen. https://www.projekt-deal.de/faqfur-teilnehmende-einrichtungen/. Zugegriffen: 16.04.2020

3. Projekt DEAL (2020) https://www.projekt-deal.de/ springer-nature-vertrag/.Zugegriffen: 16.04 .2020

4. https://www.springer.com/journal/292.Zugegriffen: 16.04 .2020

\section{Tumormutationslast als Biomarker bei Krebs: Wissenschaftler überprüfen die Leistung von sechs Gentests}

Die Menge an erworbenen Veränderungen im Erbgut im Tumorgewebe, die sogenannte Mutationslast, wird von Medizinern als Biomarker genutzt, um vorhersagen zu können, welche Patienten von einer Immuntherapie profitieren könnten. Auf dem Markt gibt es inzwischen verschiedene Tests, die durch Analyse mehrerer Hundert Gene Rückschlüsse auf diese Mutationslast zulassen. Wissenschaftler haben nun sechs Gentests auf ihre Zuverlässigkeit untersucht und konnten nachweisen, dass diese Multi-Gen-Panels sich für den Einsatz in der klinischen Routinediagnostik eignen.

Um die Mutationslast im Tumorgewebe zu bestimmen, werden aktuell vor allem zwei Verfahren eingesetzt: Bei der Exom-Sequenzierung - auch Whole-Exome Sequencing (WES) genannt - werden alle circa $20.000 \mathrm{Ge-}$ ne entschlüsselt, die für die Eiweißmoleküle in einer Zelle kodieren. „Die WES-Analyse liefert aktuell sehr genaue und verlässliche Daten, deren Auswertung und Interpretation Grundlage für weitere Therapieempfehlungen sein kann. Allerdings ist das Verfahren mit einer Analysezeit von drei bis vier Wochen relativ langsam und sehr kleine Gewebeproben sind mitunter schwierig mittels WES zu analysieren", so Stefan Fröhling, Geschäftsführender Direktor am Nationalen Centrum für Tumorerkrankungen (NCT) Heidelberg und Leiter der Abteilung für Translationale Medizinische Onkologie am Deutschen Krebsforschungszentrum (DKFZ).

Bei einer Gen-Panel-Untersuchung wird eine kleinere Anzahl von mehreren Hundert Genen in kürzerer Zeit analysiert. Die Methode lässt auf Basis der untersuchten Genabschnitte eine Schätzung der Mutationslast im Tumorgewebe zu. Auch methodisch ist das Verfahren weniger aufwändig, da im Gegensatz zur WES-Analyse kein frisches Tumormaterial benötigt wird, sondern die Untersuchung an paraffineingebetteten Gewebeschnitten erfolgen kann, wie sie üblicherweise für die Diagnose genutzt werden und in der Pathologie vorliegen. Seit Kurzem kann sogar Tumor-DNA aus dem Blut des Patienten für die Multi-Gen-Analyse verwendet werden.

Mittlerweile sind mehrere Gentests für die Bestimmung der Tumormutationslast erhältlich, die in einem Labor eingesetzt werden können. Allerdings fehlte bisher eine detaillierte Bewertung der Gesamtleistung dieser TMB(Tumor Mutational Burden)-Tests. In einer aktuellen Untersuchung haben nun
Wissenschaftler und Ärzte mehrerer Universitätspathologien in Deutschland und der Schweiz unter der Schirmherrschaft der Qualitätssicherungs-Initiative Pathologie (QuiP) die Leistung und Qualität von sechs verschiedenen Gentests an 20 Tumorproben überprüft, untereinander verglichen und der Analysegenauigkeit des WES-Verfahrens gegenübergestellt.

Die Studie hat gezeigt, dass man mit den untersuchten Gen-Panels die Tumormutationslast näherungsweise bestimmen kann und ein verlässliches Ergebnis erhält, um Patienten gezielt auswählen zu können, die von einer Immuntherapie profitieren könnten. Die Studienergebnisse sind ein wichtiger Beitrag zur Bewertung solcher Gentests in der klinischen Routinediagnostik", so M. Schlesner, Leiter der Nachwuchsgruppe Bioinformatik und Omics Data am DKFZ „Wir konnten aber auch Faktoren identifizieren, die die Ergebnisse der Gentests in der täglichen Praxis beeinflussen. Hierzu gehören beispielsweise die Anzahl der Tumorzellen im Gewebeschnitt oder auch die Qualität der enthaltenen DNA. Weitere Untersuchungen werden sich daher auch damit beschäftigen, diese Störfaktoren zu reduzieren und einheitliche bioinformatische Analyseverfahren zu entwickeln", ergänzt Stenzinger.

Literatur: Stenzinger A, Endris $\mathrm{V}$ et al (2020) Harmonization and Standardization of Panel-Based Tumor Mutational Burden (TMB) Measurement: Real-World Results and Recommendations of the QuIP StudyJournal of Thoracic Oncology (JTO). https://www.jto.org/article/S15560864(20)30135-0/pdf

Quelle: PM NCT Heidelberg, 03.03.2020 